\title{
O-GlcNAcylation in early stages of chronic lymphocytic leukemia; protocol development for flow cytometry
}

\author{
Viktória Temesfő̈i ${ }^{\mathrm{a}, \mathrm{b}}$, Kinga Molnár ${ }^{\mathrm{a}}$, Péter Kaltenecker ${ }^{\mathrm{c}}$, Barbara Réger $^{\mathrm{a}}$, Árpád Szomor ${ }^{\mathrm{d}}$, \\ Zoltán Horváth-Szalai ${ }^{\mathrm{a}}$, Hussain Alizadeh $^{\mathrm{d}}$, Béla Kajtáre ${ }^{\mathrm{e}}$, Tamás Kőszegia ${ }^{\mathrm{a}}$, , Attila Miseta $^{\mathrm{a}}$, \\ Tamás Nagy ${ }^{\mathrm{a}, *}$ and Zsuzsanna Faust ${ }^{\mathrm{a}, \mathrm{f}, *}$ \\ ${ }^{a}$ Department of Laboratory Medicine, Medical School, University of Pécs, Pécs, Hungary \\ ${ }^{\mathrm{b}}$ Lab-on-a-Chip Research Group, János Szentágothai Research Center, University of Pếs, iécs, Hungary \\ ${ }^{c}$ Laboratory of Actin Cytoskeleton Regulation, Institute of Genetics, Biological Reșec rch Centre, Eötvös Loránd \\ Research Network (ELKH), Szeged, Hungary \\ ${ }^{\mathrm{d}}$ Division of Hematology, 1st Department of Internal Medicine, Medical Schoo University of Pécs, Pécs, Hungary \\ 'Department of Pathology, Medical School, University of Pécs, Pécs, Hung ir. \\ ${ }_{\mathrm{f}}^{\mathrm{f}}$ Department of Transfusion Medicine, Medical School, University of Pécs, Yecs, Hungary
}

Received 26 October 2020

Accepted 2 June 2021

Abstract.

BACKGROUND: Recent studies proved that metaboli humges in malignant disorders have an impact on protein glycosylation, however, only a few attempts have been made so ar a ase O-GlcNAc analysis as a prognostic tool. Glucose metabolism is reported to be altered in hematological malignan res thus, we hypothesized that monitoring intracellular O-GlcNAc levels in Rai stage 0-I (Binet A) CLL patients could give at pu insights regarding subtle metabolic changes of progression which are not completely detected by the routine follow- $p$ medures.

OBJECTIVE: In this proof of concep. stuly we established a flow cytometric detection method for the assessment of OGlcNAcylation as a possible prognos ic 1 arker in CLL malignancy which was supported by fluorescence microscopy.

METHODS: Healthy volunteers and CLL patients were recruited for this study. Lymphocytes were isolated, fixed and permeabilised by various methods to $\mathrm{I}$ a $\mathrm{a}$ - ile optimal experimental condition for O-GlcNAc detection by flow cytometry. O-GlcNAc levels were measured and co $n_{\mathrm{P}}$ ared to lymphocyte count and various blood parameters including plasma glucose level.

RESULTS: The protocol ueveloped includes red blood cell lysis, formalin fixation, $0.1 \%$ Tween 20 permeabilisation and employs standardized cell number per sample and unstained controls. We have found significant correlation between O-GlcNAc levels and WBC $\left(R^{2}=0.8535, p<0.0029\right)$ and lymphocyte count $\left(R^{2}=0.9225, p<0.0006\right)$ in CLL patients. Interestingly, there was no such correlation in healthy individuals $\left(R^{2}=0.05664\right.$ for O-GlcNAc vs WBC and $R^{2}=0.04379$ for O-GlcNAc vs lymphocytes).

CONCLUSION: Analyzing O-GlcNAc changes in malignant disorders, specifically in malignant hematologic diseases such as CLL, could be a useful tool to monitor the progression of the disease.

Keywords: Chronic lymphocytic leukemia, O-GlcNAcylation, RL2, immunometabolism, flow cytometry

*Corresponding authors: Zsuzsanna Faust and Tamás Nagy, Department of Laboratory Medicine, Medical School, University of
Pécs, H-7624 Pécs, Ifjúság útja 13, Hungary. Tel.: +36 72536120 E-mail: faust.zsuzsanna@pte.hu,nagy.tamas@pte.hu. 


\section{Introduction}

Chronic lymphocytic leukemia (CLL) is the most common type of leukemia in adulthood. The incidence rate in Europe varies from 3.6/100,000 to 6.9/ 100,000 [1], while in Hungary the prevalence is 40 $50 / 100,000$ with an incidence rate of 4-5/100,000 new case/year (personal communication with the Advisory Board of the Hungarian Society of Hematology and Transfusiology). The disease is basically characterized by an indolent course, slow progression and long term survival, however, in some patients the course is rapidly progressing [2]. The current diagnostic procedure and staging of CLL involves the evaluation of routine biochemical, hematological and genetic parameters such as serum beta-2-microglobulin, lactate dehydrogenase, white blood cell count, lymphocytosis, mutation status and immunophenotyping. Also, bone marrow sample evaluation is recommended [2,3]. Although obtained data are exact, the current staging systems cannot effectively predict the progression. Determination of some crucial points such as lymph node involvement, are largely subjective and in some cases not specific to the disease. In the peripheral blood, clonally accumulated B cells seem to be quite homogenous resulting that changes of the disease cannot be timely and sensitively detected by laboratory findings. Therefore, in the follow-up of CLL it would be helpful to find an ear ly and cost-effective marker that could decipher votle changes in the disease status. Based on its r gut $^{1}$ tory role in immune cell homeostasis and activatior, measuring O-GlcNAcylation, a metabolic ac vivy-related marker, could be a promising possib: ity [4,5].

Uncontrolled proliferation in r lah gnancies requires nutrient supply in a different ra e compared to normally functioning cells. Most tyres or tumors shift their energy metabolism towards orycolytic activity even under aerobic conditions $\mathbf{k}$. 1 ich results in elevated lactate production and lower pyruvate transmission to the mitochondria. In order to compensate the less efficient production of adenosine-triphosphate (ATP) neoplastic cells upregulate glucose transporter (GLUT) expression to increase glucose uptake [6]. The activity of tumor suppressors and oncogenes which influence the response capability of the cells e.g. towards proliferation control are in a mutual relationship with the glycolytic rate and glycosyl modifications of specific proteins $[7,8]$.

The assessment of altered metabolism has diagnostic and prognostic values in several diseases.

O-GlcNAcylation is proven to be altered in certain conditions, such as type 2 diabetes mellitus [9],
Alzheimer's disease [10] and in cancer [11-14]. Leukemic B cells have been reported to differ from healthy B lymphocytes both in terms of overall intracellular glycosylation and O-GlcNAcylation of certain proteins as well $[11,15]$. Cellular metabolic preferences are associated with drug sensitivity and glycolytic activity seem to be connected to overall survival in CLL [16].

Glucose and glutamine uptake and breakdown are facilitated by the upregulation of oncogenic signaling leading to the increased production of intermediates which fuel various biosynthetic routes. Expanded flux through the hexosamine biosynthetic pathway (HBP) causes elevated uridine-diphosphate (UDP)GlcNac production thus, rasitively affects the $\mathrm{O}$ GlcNAcylation level [17, 1 . O-glycosylation is a common post-translatio al modification on proteins which occurs on the cerme and threonine side chains through the hydro yl oxygen by the addition of a $\mathrm{N}$ acetyl-galactos.me (GalNAc) or O-mannose and $\beta$ $\mathrm{N}$-acetyl-gluc samine (GlcNAc). A large percentage of the gr. cose flux through the HBP is fueling stable prote in- lycosylation while O-GlcNAc modifications c. rric d out by glycosyltransferase and glycosidase en$\triangle$ vmes are dynamically regulated by intrinsic and exirinsic factors [8,19-21].

Our focus was set on the prognostic opportunities of O-GlcNAcylation in CLL which is fundamentally related to the general metabolic status and activity of the cells. Various approaches can be found in the literature regarding the measurement of O-GlcNAcylation using flow cytometry [22-24]. We tested the use of the RL2 antibody clone to establish a simple and reliable protocol to detect the intracellular O-GlcNAcylation in lymphocytes. RL2 is a monoclonal, $\operatorname{IgG}_{1}$ isotype antibody which detects $\mathrm{O}$-linked glycoproteins in the cytoplasm and nucleus [25].

Assessing the O-GlcNAcylation level of the transformed B cells of CLL patients may give us an insight how intracellular O-GlcNAcylation can be related to disease development in the early phases where clinical classification cannot detect the subtle changes in progression yet. In our study, we examined the $\mathrm{O}$ GlcNAcylation level of lymphocytes of healthy volunteers and CLL patients by flow cytometry and microscopy using the anti-O-GlcNAcylation antibody clone RL2 in order to find the differences between normal and malignant cells and the correlation of $\mathrm{O}$ GlcNAcylation level with hematological and biochemical factors in the two groups. 


\section{Materials and methods \\ 2.1. Subjects of the study}

In each case $2 \times 3 \mathrm{~mL}$ venous blood was drawn into BD Vacutainer $K_{2} \mathrm{E}$ (K-EDTA) tubes at the same timing after overnight fasting from healthy individuals $(n=7)$ and CLL patients $(n=7)$ of Rai stage 0-I which is part of stage 'A' in Binet classification. Patients were included on the basis that their progression was slow. The followings were defined as exclusion criteria: receiving any treatment related to the investigated disease, having previous history of diabetes or other metabolic disorders. Experiments were performed using red blood cell lysed whole blood. Selected biochemical and hematological parameters were measured of each individual (white blood cell and lymphocyte count, plasma glucose, lactate, serum total protein, albumin) at the accredited laboratory of the Department of Laboratory Medicine, Clinical Center, University of Pécs, Hungary using a Cobas Integra 400 plus (Roche Gmbh, Mannheim, Germany) automated chemical analyzer and a Sysmex XN 9000 (Sysmex Corporation, Kobe, Japan) automated blood cell counter system. Immunoglobulin heavy-chain variable region (IGHV) gene mutation analysis was performed at the Department of Pathology, Clinical Center, University or Pécs, Hungary, by Sanger sequencing using lead 1 or framework region I (FRI) and JH consensus primcrs. Laboratory and patient data are listed in Si pp ementary Table 1 . The study was approved by $t^{t} / \mathrm{C}$ Regional Ethics Committee of the Medical Sch oc 1 University of Pécs in accordance with the Helsin t declaration (no. 7753-PTE2019). Healthy volurtec-c and patients were fully informed and written conson $\mathrm{t}$ was signed by each participant.

\subsection{Reagents}

Red blood cell lysis was performed using BD FACS Lysing Solution (Becton, Dickinson and Company, Franklin Lakes, NJ, USA). $10 \%$ buffered formalin (Sigma-Aldrich, Darmstadt, Germany) was used to fix the samples. Ammonium chloride $\left(\mathrm{NH}_{4} \mathrm{Cl}\right)$, methanol, Triton-X100 and Tween 20, RPMI, BSA, N-acetyl-D-glucosamine and PUGNAc were purchased from Sigma-Aldrich (Darmstadt, Germany). BD Phosflow Perm Buffer III and BD FACS Lysing Solution were from Becton, Dickinson and Company, Alexa Fluor (AF) 488 labeled anti-O-GlcNAcylation antibody (clone: RL2) and isotype control (IgG1) were from
Novus Biologicals, Alexa Fluor (AF) 546 conjugated Phalloidine (Pha) and 4', 6-diamidino-2-phenylindole (DAPI) were purchased from Thermo Fisher (Waltham, MA, USA)

\subsection{Staining protocol and flow cytometry}

White blood cell and lymphocyte counts were determined from the original blood sample tube on a Sysmex XN 9000 hematology analyzer (Sysmex Corporation, Kobe, Japan). Based on the quantitative data whole blood volume was calculated in order to get the necessary number of lymphocytes in each sample and experiment following red blood cell lysis. In experiments where PUGNAc was applied as positive control to fuel O-GlcNAcylation, cells wc ro kept in RPMI containing $50 \mu \mathrm{M}$ PUGNAc tor 1 nour at $37^{\circ} \mathrm{C}$ and $5 \% \mathrm{CO}_{2}$ in a humidified in uvat or. Fixation was performed for 20 min using $10 \%$ tormalin solution which is equivalent approximatei $\%$ \% formaldehyde. The residual fixative in ne samples was quenched by $10 \mathrm{~min}$ incubation in $10 \mathrm{~m} N$ buffered $\mathrm{NH}_{4} \mathrm{Cl}$ in phosphate buffered saline (PRS) $300 \mu \mathrm{L}$ of the tested permeabilisation reagents (C 1\% Triton X-100 or $0.1 \%$ Tween 20 or $90 \%$ methanol r BD PhosFlow Perm Buffer III) were kept on the sedimented cells for 20 minutes. Blocking was applied using 5\% BSA dissolved in phosphate buffered saline (PBS). Samples were washed between each step with $2 \mathrm{ml}$ PBS solution and centrifuged at $600 \mathrm{~g}$ for $5 \mathrm{~min}$. Labeling was performed either with RL2 antibody or in certain experiments with isotype control for $60 \mathrm{~min}$ at room temperature. Antibodies were used at $1 \mu \mathrm{g} / \mathrm{mL}$ working concentration in each experiment. In the specificity evaluation experiments as a negative control, RL2 antibodies were incubated for $30 \mathrm{~min}$ in $20 \mathrm{mM} \mathrm{N}$ acetyl-D-glucosamine prior adding them to the cells. Following the staining procedure samples were washed with permeabilisation reagent containing PBS to prevent the unbound antibodies from being trapped within the cells [26]. During optimization processes experiments were measured in three technical replicates. A minimum of 10.000 events were collected from each sample. Measurements were carried out on a BD FACS Canto II instrument (Becton, Dickinson and Company, Franklin Lakes, NJ, USA). Flow cytometry gating strategy included a time gate in which flow stability was checked. Doublet discrimination was performed based on forward scatter height and area (FSC-H/FSC-A). Lymphocyte population was identified in a side scatter/forward scatter area (SSC-A/FSC-A) gate. Gating strategy is demonstrated in Supplementary Fig. 1. Me- 

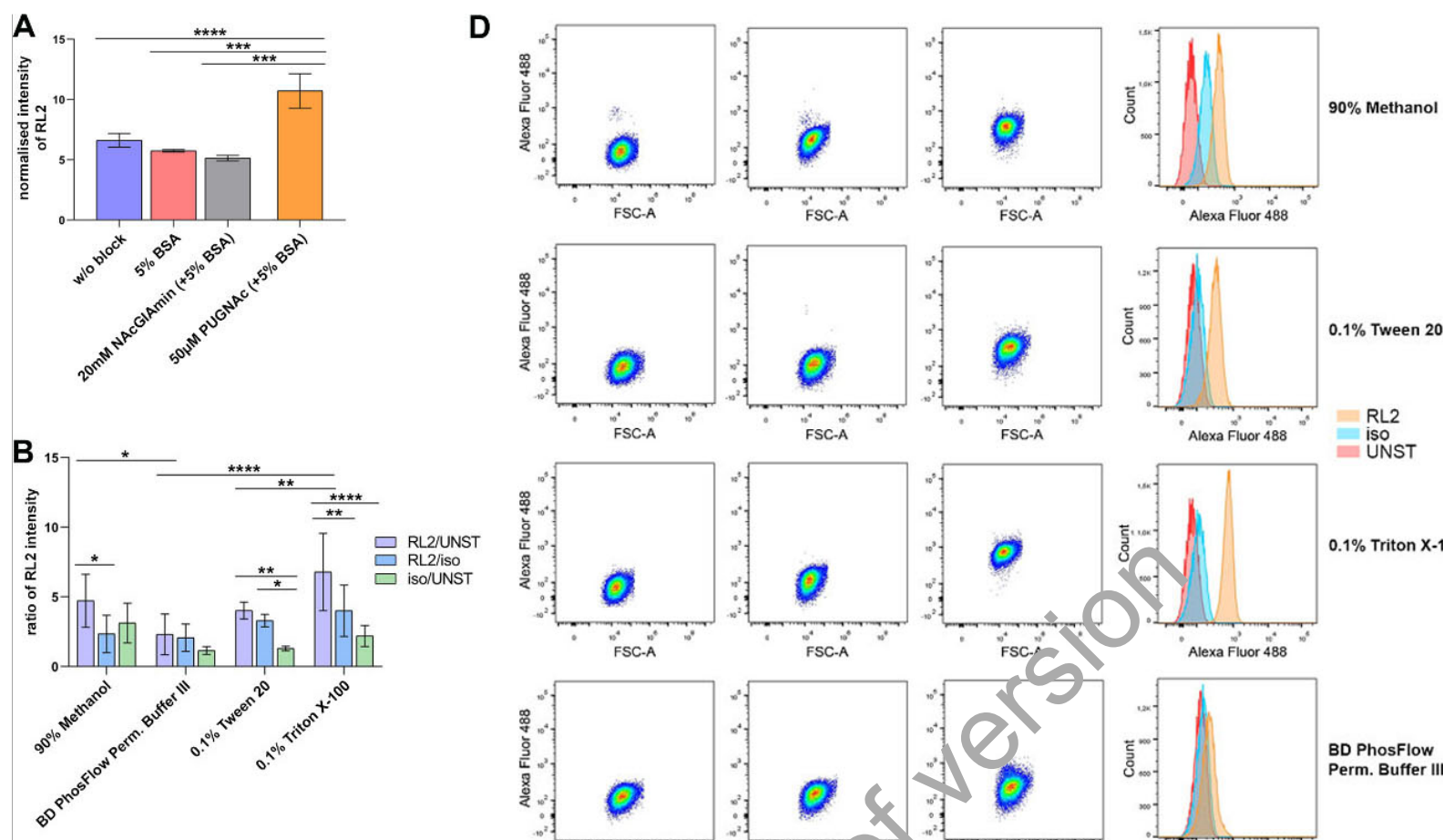

$0.1 \%$ Tween 20
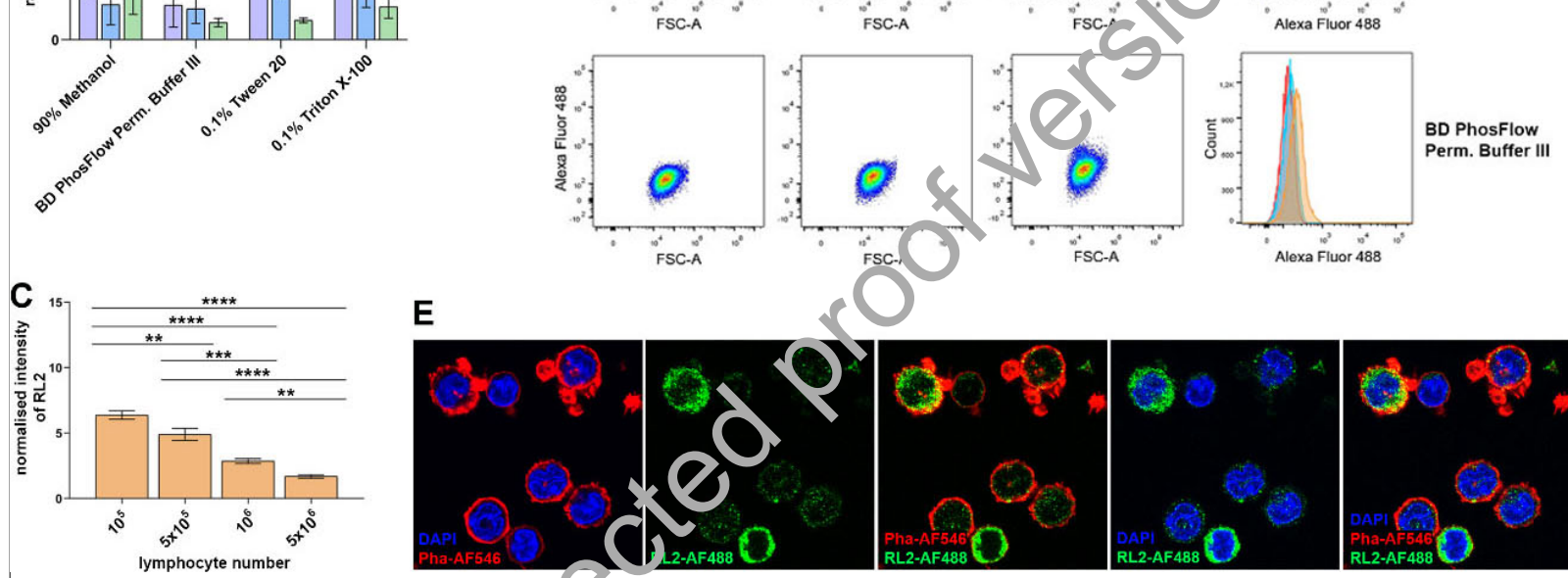

E
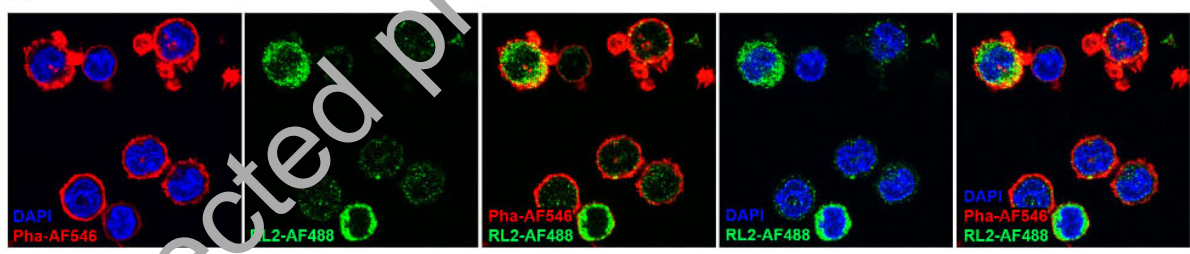

Fig. 1. Summary of the protocol validation of $-\mathrm{O}-\mathrm{GlcNAcylation}$ staining using RL2 antibody clone. A. Blocking and specificity. Cells were fixed with $10 \%$ formalin for $20 \mathrm{~min}$, permeab 'ised using $0.1 \%$ Tween 20 for $20 \mathrm{~min}$, RL2 concentration was $1 \mu \mathrm{g} / \mathrm{mL}$ ( $60 \mathrm{~min}$ staining). For positive control we used PUGNAc treatme it $(\$) \mu \mathrm{g} / \mathrm{mL}, 1$-hour incubation), N-acetyl-D-glucosamine served as negative control. Median intensity $( \pm \mathrm{SD})$ values included are normalize to th autofluorescent control. Ordinary one-way ANOVA, Tukey's post hoc test, three technical replicates. B. Comparison of the permeabilis ... an a gents. Cells were fixed with $10 \%$ formalin for 20 min, we tested 90\% methanol, BD PhosFlow Perm Buffer, $0.1 \%$ Tween 20 and $0.1 \%$ riton X-100 reagents for permeabilisation (20 minutes). RL2 and isotype control concentration was $1 \mu \mathrm{g} / \mathrm{mL}$ (60 min staining). Bars represent 1 tios of median intensities of different samples. Two-way ANOVA, Tukey's multiple comparison test, replicates represent six different individuars. C. Optimization of the lymphocyte number. Cells were fixed with $10 \%$ formalin for 20 min, permeabilised using $0.1 \%$ Tween 20 for $20 \mathrm{~min}$, RL2 concentration was $1 \mu \mathrm{g} / \mathrm{mL}$ (60 min staining). Median intensity values included are normalized to the autofluorescent control. One-way ANOVA, Tukey's post hoc test, three technical replicates. D. Representative figure of the permeabilisation with each reagent. Plots and histograms are generated from the fluorescent intensity of RL2-AF488 in the lymphocyte population. Cells were fixed with $10 \%$ formalin for 20 min, permeabilised using 90\% methanol, BD PhosFlow Perm Buffer, 0.1\% Tween 20 or $0.1 \%$ Triton X-100 for 20 min, RL2 concentration was $1 \mu \mathrm{g} / \mathrm{mL}$ (60 min staining). E. Confocal microscopic visualization of the performance of the finalized protocol. Blue color shows DAPI staining, RL2-AF488 is green, red color indicates Pha-AF546 staining. ${ }^{*} p<0.05,{ }^{*} p<0.01,{ }^{*} * *<0.001,{ }^{*} * * * p<0.0001$.

dian intensity values of the AF488 conjugated antibodies measured on the fluorescein isothiocyanate (FITC) channel were exported from the analysis software. Values were normalized to the unstained autofluorescent control of each individual, which went through the same procedures as the labeled samples, but the antibody was omitted from the staining solution. Analysis was carried out using FlowJo software version 10 (Becton, Dickinson and Company, Franklin Lakes, NJ, USA).

\subsection{Microscopy}

In the microscopic experiments we used the same staining protocol as for the flow cytometry regarding the 

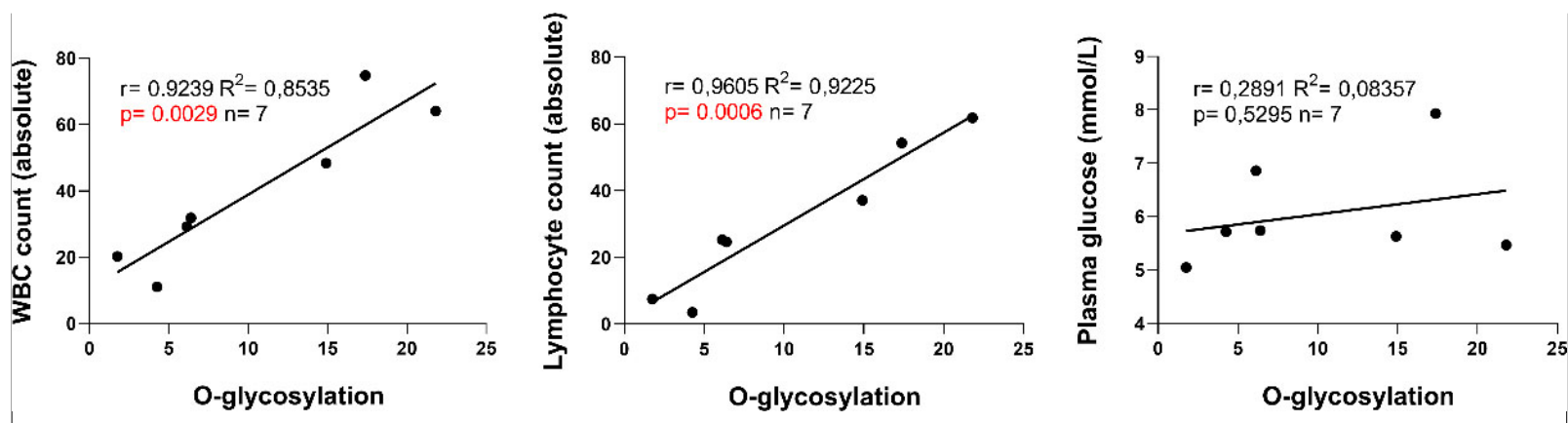

Fig. 2. Correlation of O-GlcNAcylation with white blood cell count, absolute lymphocyte number and plasma glucose level in CLL patients Pearson correlation and simple linear regression, alpha $=0.05, p$ (two-tailed), $n=7$.

O-GlcNAcylation (10\% formalin fixation, $0.1 \%$ Tween 2409 acetyl-D-glucosamine for negative control and O-(2-

20 permeabilisation buffer, blocking with 5\% BSA, $1 \mu \mathrm{g} / \mathrm{mL}$ concentration of the RL2 antibody, $60 \mathrm{~min}$ staining). In addition, filamentous actin cytoskeleton was labeled by Pha-AF546 and nuclei were stained with DAPI [27]. Confocal images of lymphocytes were captured on an Olympus IX83 inverted microscope with the CSU-W1 Spinning Disk Imaging System, equipped with Zyla sCMOS cameras (Visitron Systems GmbH, Puchheim, Germany). Representative images were selected, and brightness/contrast levels of the channels were set by using ImageJ software.

\subsection{Analysis and software}

In order to compare the effect of permeahi saion reagents and initial cell number on the stain ing and to assess antibody specificity and blocking eff ciency in the setup phase, we used ordinary ne and two-way ANOVA with Tukey's multiple On parisons test. To reveal the relationship betwee rausiatory parameters and the level of O-GlcNAcy ation of the lymphocyte population we performed Puarson correlation analysis (due to parametric data, ar d simple linear and binary logistic regression. Overall lymphocyte glycosylation level of the healthy and the CLL group was compared using unpaired $t$ test with Welch's correction. Statistical analysis was carried out in Prism 8 (GraphPad Software, San Diego, CA, USA).

\section{Results}

\subsection{Validation of the experimental protocol}

First we investigated RL2 antibody specificity and blocking efficiency of bovine serum albumin (BSA) (Fig. 1A). We chose to inhibit antibody binding by NAcetamido-2-deoxy-D-glu op ranosylidenamino) $\mathrm{N}$ phenylcarbamate (PUGN Ac) treatment for positive control. Using BSA we detucled a slight decrease in intensity compared to the res licates where no blocking was applied. Addit o. of PUGNAc resulted in significant elevation of 0 - CicNAcylation. Comparing PUGNAc treated cells with the replicates where RL2 antibodies we $\mathrm{D}$ ound by $\mathrm{N}$-acetyl-D-glucosamine prior to labelin the median fluorescence intensity of RL2 was ai no, thalf of the PUGNAc supplemented cells.

Various permeabilisation reagents were tested to assess the effect of permeabilisation on non-specific binding. We compared $90 \%$ methanol, BD PhosFlow Perm Buffer III, $0.1 \%$ Tween-20 and $0.1 \%$ Triton X-100 (Fig. 1B and D). Although 90\% methanol seems to work with RL2 staining, it turned out to be too harsh on CLL samples and lowered the final cell number at least by $40-60 \%$ by damaging the lymphocytes (Supplementary Fig. 2). The intensity ratio of RL2/unstained (UNST) samples permeabilised with BD PhosFlow Perm Buffer III was 2.302 with a standard deviation (SD) of 1.461. In contrast, $0.1 \%$ Triton $\mathrm{X}-100$ or $0.1 \%$ Tween 20 showed a significantly higher intensity ratio $(4.020 \pm 0.602$ and $6.786 \pm 2.774$, respectively). Since there was no significant difference between the RL2 versus UNST and RL2 versus isotype control (iso) samples and iso/UNST ratio was lower $(1.295 \pm 0.1475)$ than using $0.1 \%$ Triton X-100 (2.190 \pm 0.6906$)$ we chose to proceed with $0.1 \%$ Tween 20 subsequently.

As a next step, the effect of antibody concentration per lymphocyte number was tested (Fig. 1C). Instead of increasing the RL2 antibody concentration on a constant cell number we applied a less concentrated dilution of the antibody, optimized the conditions regarding blocking and permeabilisation and titrated the initial lymphocyte number using the same RL2 concentration (1 $\mu \mathrm{g} / \mathrm{mL}$ in $50 \mu \mathrm{L}$ volume). Under these conditions the 
6 V. Temesfói et al. / O-GlcNAcylation in early stages of chronic lymphocytic leukemia; protocol development for flow cytometry
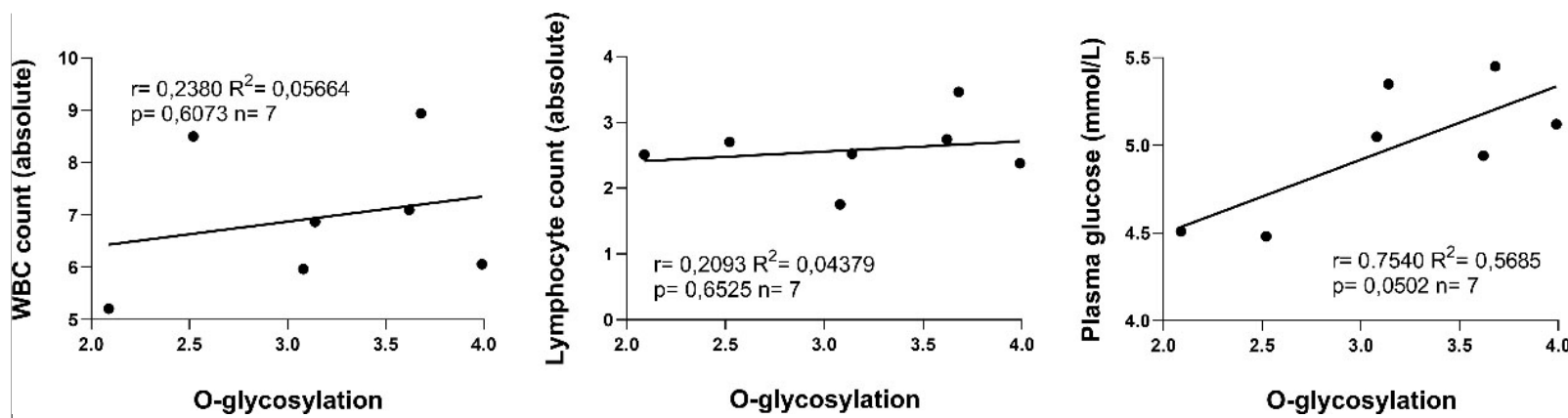

Fig. 3. Correlation of O-GlcNAcylation with white blood cell count, absolute lymphocyte number and plasma glucose level in healthy individuals Pearson correlation and simple linear regression, alpha $=0.05, p$ (two-tailed), $n=7$.

highest RL2 fluorescence intensity was achieved when the lymphocyte number was $10^{5}$.

In order to check the intracellular localization of the RL2 antibody, immunofluorescence analysis was performed using the same, flow cytometry-optimized staining conditions detailed above. Additionally, Pha-AF546 staining was used to label the actin cytoskeleton as a cell body marker and nucleus was visualized by DAPI dye (Fig. 1E). The confocal cross-section images show the intracellular distribution of RL2. The antibody is highly enriched in the cytoplasm, while a weaker, uneven signal is seen in the nucleus.

\subsection{O-GlcNAcylation level of lymphocyte populatic $n$} correlates with absolute white blood cell and lymphocyte count in early phases of CLI

A well-known characteristic of CLL is the elevation of lymphocyte number caused by the crenal expansion of neoplastic B cells over time. We ind that the normalized intensity of RL2 antibc ay and consequently the level of O-GlcNAcylation is in positive correlation with lymphocyte and total white blood cell count in CLL. We have also found tha is not associated with the actual plasma glucose level of the patient (Fig. 2). An important addition to our finding is that this positive correlation is observed within stage Rai 0-I (Binet 'A'), which suggests that the level of O-GlcNAcylation may indicate the progression of the disease while the clinical classification still remains unaltered. We could not find any relationship between O-GlcNAcylation of lymphocytes and the level of plasma lactate, serum total protein and albumin, age of the patient and elapsed years since diagnosis (Supplementary Fig. 3).

In healthy individuals, no such association could be revealed regarding total white blood cell and lymphocyte count (Fig. 3). Fasting glucose level was found to be also unrelated to O-GlcNAcylation, however it seems
279

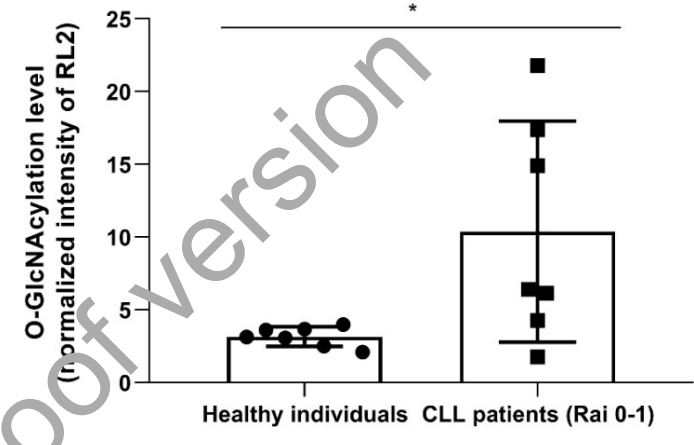

g. 4. Comparison of O-GlcNAcylation level of lymphocytes in ealthy individuals and in CLL patients of Rai stage 0 and I (Bine A). Bars represent normalized median intensity $( \pm$ SD). Unpaired $t$ test with Welch's correction, $p$ (two tailed) $=0.0457, n=14$. SD of CLL patients $=7.595, \mathrm{SD}$ of healthy individuals $=0.6748$.

315 that in this group there is a trend that higher plasma glucose concentration is accompanied with slightly higher level of intracellular O-GlcNAcylation without statistical significance (Fig. 3). No further association was revealed regarding the included hematological and biochemical parameters in the healthy control group (Supplementary Fig. 4).

CLL patients showed significantly higher O-GlcNAcylation rate of the lymphocyte population compared to healthy individuals and standard deviation (SD) was also higher in this group (Fig. 4). Patients with more advanced lymphocyte proliferation within stage Rai 0-I turned out to have elevated O-GlcNAcylation level. We found a linear relationship between the white blood cell, lymphocyte count and O-GlcNAcylation. The higher the number of white blood cells, lymphocytes; the higher the level of O-GlcNAcylation.

\section{Discussion}

Given the recent therapeutic improvements and the discovery of independent prognostic markers, the tradi- 
tional clinical staging systems of CLL tend to be insufficient in defining more than three prognostic groups [3]. Unlike most of the neoplastic diseases, patients with low risk CLL do not benefit from early therapeutic interventions [2,3], however our expanding knowledge about immunometabolism gives the opportunity to look deeper into the ongoing processes, resulting in discovering the prognostic and therapeutic role of metabolism related markers.

A useful biomarker in any diagnostic considerations requires two criteria; the first is that it is present (i.e. detectable) in a certain condition and only in this condition. In other words, high or at least acceptable sensitivity and specificity. The second is that it should precede the particular condition. Our data are promising in respect to the first criteria and we expect that OGlcNAc elevation precedes the progression of CLL in the early stages. Findings in previous studies indicate that metabolic alterations and consequently O-GlcNAc changes are indeed part of the progress and are associated with worse prognosis $[7,8]$. In our study we intended to test whether the measurement of the overall cellular level of O-GlcNAcylation can be integrated into the routine follow-up of CLL.

Accurate sensitivity and specificity calculations regarding O-GlcNAcylation as a potential biomarker can only be done when the cut-off values for WBCs/lympt. ocytes are set correctly based on the results of a lar re cohort study. Since it is possible that O-GlcNAcy an is elevated in some physiological conditions a $w_{\mathrm{N}} \mathrm{ll}$, not tested here, and development of the disene, a later stages of CLL in the study of Shi and roagues [11], may also influence the level, it woul be wise to set up group-specific cut-offs and evalua te tie data separately for each group. Obviously, pro spective clinical studies are needed to be performen evaluate the predictive value of O-GlcNAc analy is.

Mass spectrometry ha oeen the main approach to identify O-GlcNAc modification sites and to reveal the stoichiometric features so far. Although large proteome datasets are available with valuable information on the mapping sites of glycosyl side chains, more functional analyses are needed which can be easily integrated into the clinical diagnostics as well [10]. Western blot is a technical approach which has been implemented so far in the detection of post-translational modifications, although it is not an optimal workflow to be included in clinical procedures [28]. Immunohistochemistry, flow cytometry or other fluorescence based methods serve as a good basis to rapid diagnostics [29].

In order to employ O-GlcNAc detection in clinical laboratory diagnostics, a rapid and high through-
38355 put method has to be developed. Taking into account the methodological considerations, such as rapidity and accuracy and the specific requirements of intracellular labeling, flow cytometry may be an optimal approach to be included into the routine procedures [30]. However, some main points have to be acknowledged when investigating such a dynamically changing post-translational modification. Performing the experiments, we paid special attention to several factors which could influence the level of O-GlcNAcylation during the pre-analytical and analytical phases. Samples were processed immediately, within approx. $10 \mathrm{~min}$ following blood collection. Steps before formalin fixation were carried out at $4^{\circ} \mathrm{C}$ and on ice to prevent ex vivo metabolic changes in the cells. Red blood cell iy cis was chosen over the sucrose gradient based lympirocyte separation method so the cells got into the fir ve as soon as possible. We normalized intensity wal ues of O-GlcNAc fluorescence to the values of the same channel measured from the fluorescence $\mathrm{m}, \mathrm{u}$ one (FMO) control. In our case unstained cell , hich were treated the same way as the labeled amples, except antibody staining, served as FMC co ntrols. Although isotype control is a separate e) pe mental configuration than a relevant control for unspecific binding, in these circumstances we decided to trust the ratios calculated with isotype intensity to be part of the estimation of antibody adherence and to test whether intracellular adherence could be influenced by the permeabilisation.

We chose patients with favorable conditions who have slowly developing disease and have not got any treatment yet. With our flow cytometric method we could demonstrate significant differences between the O-GlcNAc levels of lymphocytes of healthy controls and CLL patients.

Our results complete the findings of Shi and colleagues who examined the level of O-GlcNAcylated proteins in CLL cells and normal peripheral blood mononuclear cells (PBMC) with immunoblotting [11]. Additionally, in our study O-GlcNAcylation turned out to be positively correlating with lymphocyte and total white blood cell count in CLL patients. However, it should be noted that cases only from the Rai 0-I stages (Binet 'A') were included and patients from later stages were not investigated. Results also showed that CLL patients have lower general O-GlcNAcylation in the lymphocyte population when lymphocyte count falls within or approaches the normal range. This raises the possibility that an increase in O-GlcNAcylation of specific proteins is associated with active proliferation [31,32].

O-GlcNAcylation is considered to be a nutrient sensing mechanism which has regulatory role in epigenetic 
and genetic processes [21]. Whereas the clonal expansion in CLL requires active proliferation, circulating cells seem to have an immunologically quiescent phenotype which comes with resistance to apoptotic signals. For example, hyper-O-glycosylation of the NF-KB transcription factor family prevents the tumor cells from apoptosis by keeping the transcription of target genes constitutively active [16]. From another point of view, since hyper-O-GlcNAcylation of apoptotic pathways is reported to elevate their activity [31,32], it is also possible that increased proliferation in the early stages is associated with increased apoptosis thus, elevating cell number does not necessarily mean the absence of any control and the current condition of the disease can be maintained for a long time without any intervention (watchful waiting) [33].

O-GlcNAcylation has been also reported to reflect the disease severity in leukemia through stages. Developing high-risk cytogenetic abnormalities or reaching an advanced condition which requires therapeutic intervention resulted in the decrease of O-GlcNAc modifications in the later stages (Rai II-IV) in the study of Shi and colleagues [11]. Our findings might give an addition to the prognostic use of O-GlcNAcylation in the early phases (Rai 0-I).

There may be several reasons to elucidate why $\mathrm{O}$ GlcNAcylation increases in direct proportion to c 11 number in the first period of the disease and the itecreases in later stages regardless of clonal expnsion. Studies using next generation sequencing re vea ed the genetic complexity of CLL which incluce ofveral mutations and copy number alteration which are accumulated during progression [34 $\mathrm{m}$, are related to metabolic regulation, such as $T P 55[25]$ and $A T M$ or the IGHV mutation status itself $[16]$. Besides the overall alterations, changes of $\mathrm{O}$ sirNAcylation in the course of the disease may invoive specific proteins of certain signaling routes $[15,36,37]$.

The requirements of our developed protocol is comparable with routinely used phenotyping procedures for flow cytometry. Using our method, we could demonstrate significant differences between O-GlcNAcylation levels of lymphocytes of healthy control persons and CLL patients and revealed the correlation between lymphocyte count and lymphocyte O-GlcNAcylation in the early stages of CLL.

Although we cannot rule out that elevation of case number would affect the results, the fact that correlation of O-GlcNAcylation and lymphocyte number was proven within a homogenous IGHV mutated group, suggests that glycosylation as a marker for disease pro-
49639 gression may be used in the mutated group or independently from mutation status where disease course is less severe and it is challenging to obtain information about the progression. Including metabolic parameters of the tumor cells into the diagnostic and follow-up processes of CLL could be a great advantage regarding the estimation of drug sensitivity as well [16]. Given its link to the glycolytic activity of the cells, monitoring the changes of O-GlcNAcylation during progression could have an impact on the choice of therapeutic intervention by completing our knowledge about the behavior of the neoplastic cells, besides the already used markers such as lymphocyte doubling time, CD38 and ZAP-70 expression [38]. Since glycolytic activity contributes to drug resistan $\mathrm{e}$ in CLL [16], measuring O-GlcNAcylation could 'ete stimate the potential vulnerability of the patient ro ungs having association with metabolic activities GLL.

\section{Cor clusiv.s}

Ou p oof of concept study demonstrated for the first tine hat O-GlcNAcylation of the lymphocyte populaion in the early stages of CLL (Rai 0-I) is in correlation with total white blood cell and lymphocyte count. We have also shown that detection of this post-translational modification seems to be a potential candidate to be included into the routine follow-up procedures of this disease.

\section{Acknowledgments}

This work was supported by grants from University of Pécs, Medical School, KA-2018-17; KA-2018-21; KA-2019-36; KA-2019-28 and EFOP 3.6.1-16-201600004 (Comprehensive Development for Implementing Smart Specialization Strategies at the University of Pécs); GINOP-2.3.2-15-2016-00021; and Higher Education Institutional Excellence Program of the Ministry for Innovation and Technology in Hungary, within the framework of the second thematic program of the University of Pécs (FIKP II.2).

\section{Author contributions}

Conception: Zs.F., V.T. and T.N.

Interpretation and analysis of data: V.T., Zs.F. and K.M. Preparation of the manuscript: V.T., P.K., T.N., Zs.F. 
Revision for important intellectual content: T.K., Zs.F., 5982 [12] T.N., P.K., H.A., Á. Sz., B.R., B.K., Z.H. Sz.

Supervision: Zs.F., T.N., T.K., A.M. All authors have read and agreed to the published version of the manuscript.

\section{Conflict of interest}

The authors declare no conflict of interest.

\section{Supplementary data}

The supplementary files are available to download from http://dx.doi.org/10.3233/CBM-203049.

\section{References}

[1] D. Jeyakumaran, A. Kempel and S. Cote, An assessment of the number of chronic lymphocytic leukemia (CLL) patients eligible for front-line treatment but unsuitable for full-dose fludarabine across the european union, Value in Health 19 (2016), 574-575.

[2] M. Hallek, B.D. Cheson, D. Catovsky, F. Caligaris-Cappio, G Dighiero, H. Döhner, P. Hillmen, M.J. Keating, E. Montserrat, K.R. Rai, T.J. Kipps and I.W.o.C.L. Leukemia, Guidelines to the diagnosis and treatment of chronic lymphocytic leuken ia: A report from the international workshop on chronic ly mocytic leukemia updating the national cancer institut. we ing group 1996 guidelines, Blood 111 (2008), 5446- 0456.

[3] M. Hallek, Chronic lymphocytic leukemia: 2026 pdate on diagnosis, risk stratification and treatmen , n J Hematol 94 (2019), 1266-1287.

[4] Y.H. Chang, C.L. Weng and K.I. Lim, GlcNAcylation and its role in the immune system, J Bi. mes Sci 27 (2020), 57.

[5] T. de Jesus, S. Shukla and P. Ran akrishnan, Too sweet to resist: Control of immune cell unction by O-GlcNAcylation, Cell Immunol 333 (2018, 8.-92.

[6] D. Hanahan and R. Wo nbe g, Hallmarks of cancer: The next generation, Cell 144 (2011), 646-674.

[7] R.J. DeBerardinis and N.S. Chandel, Fundamentals of cancer metabolism, Sci Adv 2 (2016), e1600200.

[8] J. Munkley and D.J. Elliott, Hallmarks of glycosylation in cancer, Oncotarget 7 (2016), 35478-35489.

[9] C. Springhorn, T.E. Matsha, R.T. Erasmus and M.F. Essop, Exploring leukocyte O-GlcNAcylation as a novel diagnostic tool for the earlier detection of type 2 diabetes mellitus, $J$ Clin Endocrinol Metab 97 (2012), 4640-4649.

10] R.J. Copeland, G. Han and G.W. Hart, O-GlcNAcomics Revealing roles of O-GlcNAcylation in disease mechanisms and development of potential diagnostics, Proteomics Clin Appl 7 (2013), 597-606.

[11] Y. Shi, J. Tomic, F. Wen, S. Shaha, A. Bahlo, R. Harrison, J.W. Dennis, R. Williams, B.J. Gross, S. Walker, J. Zuccolo, J.P. Deans, G.W. Hart and D.E. Spaner, Aberrant O-GlcNAcylation characterizes chronic lymphocytic leukemia, Leukemia $\mathbf{2 4}$ (2010), 1588-1598.
G. Fuentes-García, M.C. Castañeda-Patlán, A.S. Vercoutter Edouart, T. Lefebvre and M. Robles-Flores, GlcNAcylation is involved in the regulation of stem cell markers expression in colon cancer cells, Front Endocrinol (Lausanne) 10 (2019), 289.

[13] C.M. Ferrer, V.L. Sodi and M.J. Reginato, O-GlcNAcylation in cancer biology: Linking metabolism and signaling, $J \mathrm{Mol}$ Biol 428 (2016), 3282-3294.

[14] X. Zhang, Y. Gu, W. Yu and H. Liang, Regulation and clinica significance of O-GlcNAc transferase in cancer, Oncotarget (2017).

[15] B. Zhang, P. Zhou, X. Li, Q. Shi, D. Li and X. Ju, Bitterness in sugar: O-GlcNAcylation aggravates pre-B acute lymphocytic leukemia through glycolysis via the PI3K/Akt/c-Myc pathway, Am J Cancer Res 7 (2017), 1337-1349.

[16] J. Lu, M. Böttcher, T. Walther, D. Mougiakakos, T. Zenz and W. Huber, Energy metabolism is co-determined by genetic variants in chronic lymphocytic leukemia and influences drug sensitivity, Haematologica 04 (3019), 1830-1840.

[17] A. Silsirivanit, Glycos lat markers in cancer, Adv Clin Chem 89 (2019), 189-?

[18] Z. Ma and K. Vos se $\mathrm{e}^{1 \mathrm{r}} \mathrm{r}$, Cancer metabolism and elevated $\mathrm{O}$ GlcNAc in on genta signaling, J Biol Chem 289 (2014), 34457-344\% 0 .

[19] K. Ohtsut o nd J.D. Marth, Glycosylation in cellular mecha nicms of ' + alth and disease, Cell 126 (2006), 855-867.

[20] K. osseller, K. Sakabe, L. Wells and G.W. Hart, Diverse eg lation of protein function by O-GlcNAc: A nuclear and vir plasmic carbohydrate post-translational modification, $\mathrm{Curr}$ Opin Chem Biol 6 (2002), 851-857.

21] T. Nagy, V. Fisi, D. Frank, E. Kátai, Z. Nagy and A. Miseta, Hyperglycemia-induced aberrant cell proliferation; A metabolic challenge mediated by protein O-GlcNAc modification, Cells 8 (2019).

[22] D. Jayakumar, D.D. Marathe and S. Neelamegham, Detection of site-specific glycosylation in proteins using flow cytometry, Cytometry A 75 (2009), 866-873.

[23] C. Batisse, J. Marquet, A. Greffard, J. Fleury-Feith, M.C. Jau rand and Y. Pilatte, Lectin-based three-color flow cytometric approach for studying cell surface glycosylation changes that occur during apoptosis, Cytometry A 62 (2004), 81-88.

[24] S.A. Madsen-Bouterse, Y. Xu, H.R. Petty and R. Romero, Quantification of O-GlcNAc protein modification in neutrophils by flow cytometry, Cytometry A 73 (2008), 667-672.

[25] C.M. Snow, A. Senior and L. Gerace, Monoclonal antibodies identify a group of nuclear pore complex glycoproteins, $\mathrm{J} \mathrm{Cell}$ Biol 5 (1987), 1143-1156.

[26] K. Groeneveld, J.G. te Marvelde, M.W. van den Beemd, H. Hooijkaas, J.J. van Dongen, Flow cytometric detection of intracellular antigens for immunophenotyping of normal and malignant leukocytes, Leukemia 10(8) (1996), 1383-1389.

[27] V. DesMarais, R.J. Eddy, V.P. Sharma, O. Stone, J.S. Condeelis, Optimizing leading edge F-actin labeling using mul tiple actin probes, fixation methods and imaging modalities Biotechniques 66(3) (2019), 113-119.

[28] J.J. Bass, D.J. Wilkinson, D. Rankin, B.E. Phillips, N.J. Szewczyk, K. Smith and P.J. Atherton, An overview of technical considerations for Western blotting applications to physiological research, Scand J Med Sci Sports 27 (2017), 4-25.

[29] C.H. Dunphy, Applications of flow cytometry and immunohistochemistry to diagnostic hematopathology, Arch Pathol Lab Med 128 (2004), 1004-1022.

[30] T. Nagy, E. Kátai, V. Fisi, T.T. Takács, A. Stréda, I. Wittmann and A. Miseta, Protein O-GlcNAc modification increases in 
10 V. Temesfói et al. / O-GlcNAcylation in early stages of chronic lymphocytic leukemia; protocol development for flow cytometry

white blood cells after a single bout of physical exercise, Front $\quad 6624$ Immunol 9 (2018), 970

[31] D.H. Lee, N.E. Kwon, W.J. Lee, M.S. Lee, D.J. Kim, J.H. Kim and S.K. Park, Increased O-GlcNAcylation of c-Myc promotes Pre-B cell proliferation, Cells 9 (2020).

32] M. Jiang, Z. Qiu, S. Zhang, X. Fan, X. Cai, B. Xu, X. Li, J. Zhou, X. Zhang, Y. Chu, W. Wang, J. Liang, T. Horvath, X. Yang, K. Wu, Y. Nie and D. Fan, Elevated O-GlcNAcylation promotes gastric cancer cells proliferation by modulating cell cycle related proteins and ERK 1/2 signaling, Oncotarget 7 (2016), 61390-61402.

[33] J. Evans, S. Ziebland and A.R. Pettitt, Incurable, invisible and inconclusive: Watchful waiting for chronic lymphocytic leukaemia and implications for doctor-patient communication, Eur J Cancer Care (Engl) 21 (2012), 67-77.

[34] G. Gaidano, R. Foà and R. Dalla-Favera, Molecular patho- genesis of chronic lymphocytic leukemia, J Clin Invest 122 (2012), 3432-3438.

[35] M. Lacroix, R. Riscal, G. Arena, L.K. Linares and L. Le Cam Metabolic functions of the tumor suppressor p53: Implications in normal physiology, metabolic disorders, and cancer, $M o$ Metab 33 (2020), 2-22.

[36] T.Y. Chou, C.V. Dang and G.W. Hart, Glycosylation of the c-Myc transactivation domain, Proc Natl Acad Sci USA 92 (1995), 4417-4421.

[37] R.M. de Queiroz, E. Carvalho and W.B. Dias, O-GlcN Acylation: The sweet side of the cancer, Front Oncol 4 (2014) 132.

[38] C.I. Amaya-Chanaga and L.Z. Rassenti, Biomarkers in chronic lymphocytic leukemia: Clinical applications and prognostic markers, Best Pract Res Clin Haematol 29 (2016), 79-89. 